\title{
Non-commutative multivariable Reidemester torsion and the Thurston norm
}

\author{
SHELlY HARVEY \\ STEFAN FRIEDL
}

Given a 3-manifold the second author defined functions $\delta_{n}: H^{1}(M ; \mathbb{Z}) \rightarrow \mathbb{N}$, generalizing McMullen's Alexander norm, which give lower bounds on the Thurston norm. We reformulate these invariants in terms of Reidemeister torsion over a noncommutative multivariable Laurent polynomial ring. This allows us to show that these functions are semi-norms.

57M27; 57N10

\section{Introduction}

Let $M$ be a 3-manifold. Throughout the paper we will assume that all 3-manifolds are compact, connected and orientable. Let $\phi \in H^{1}(M ; \mathbb{Z})$. The Thurston norm of $\phi$ is defined as

$$
\|\phi\|_{T}=\min \left\{\chi_{-}(S) \mid S \subset M \text { properly embedded surface dual to } \phi\right\},
$$

where for a surface $S$ with connected components $S_{1}, \ldots, S_{k}$ we write $\chi_{-}(S)=$ $\sum_{i=1}^{k} \max \left\{0,-\chi\left(S_{i}\right)\right\}$. We refer to Thurston [18] for details.

Generalizing work of Cochran [1], the second author introduced in [7] a function

$$
\delta_{n}: H^{1}(M ; \mathbb{Z}) \rightarrow \mathbb{N}_{0} \cup\{-\infty\}
$$

for every $n \in \mathbb{N}$ and showed that $\delta_{n}$ gives a lower bound on the Thurston norm. These functions are invariants of the 3-manifold and generalize the Alexander norm defined by $\mathrm{C}$ McMullen in [11]. We point out that the definition we use in this paper differs slightly from the original definition when $n=0$ and a few other special cases. We refer to Section 4.3 for details.

The relationship between the functions $\delta_{n}$ and the Thurston norm was further strengthened in Harvey [8] (cf also Cochran [1] and Friedl [4]) where it was shown that the 
$\delta_{n}$ give a never decreasing series of lower bounds on the Thurston norm, ie for any $\phi \in H^{1}(M ; \mathbb{Z})$ we have

$$
\delta_{0}(\phi) \leq \delta_{1}(\phi) \leq \delta_{2}(\phi) \leq \cdots \leq\|\phi\|_{T} .
$$

Furthermore it was shown in Friedl-Kim [5] that under a mild assumption these inequalities are an equality modulo 2 .

In his original paper [18], Thurston showed that $\|-\|_{T}$ is a semi-norm. It is therefore a natural question to ask whether the invariants $\delta_{n}$ are semi-norms as well. In [7] this was shown to be the case for $n=0$. The following theorem, which is a special case of the main theorem of this paper (cf Theorem 4.2), gives an affirmative answer to this question for $n \geq 1$.

Theorem 1.1 Let $M$ be a 3-manifold with empty or toroidal boundary. Assume that $\delta_{n}(\phi) \neq-\infty$ for some $\phi \in H^{1}(M ; \mathbb{Z})$, then

$$
\delta_{n}: H^{1}(M ; \mathbb{Z}) \rightarrow \mathbb{N}_{0}
$$

is a semi-norm.

In particular, this allows us to show that the sequence $\left\{\delta_{n}\right\}$ is eventually constant. That is, there exists an $N \in \mathbb{N}$ such that $\delta_{n}=\delta_{N}$ for all $n \geq N$ (cf Proposition 4.4).

Before we address whether the $\delta_{n}$ are norms, we discuss a more algebraic problem. Recall that given a multivariable Laurent polynomial ring $\mathbb{F}\left[t_{1}^{ \pm 1}, \ldots, t_{m}^{ \pm 1}\right]$ over a commutative field $\mathbb{F}$ we can associate to any non-zero $f=\sum_{\alpha \in \mathbb{Z}^{m}} a_{\alpha} t^{\alpha} \in \mathbb{F}\left[t_{1}^{ \pm 1}, \ldots, t_{m}^{ \pm 1}\right]$ a semi-norm on hom $\left(\mathbb{Z}^{m}, \mathbb{R}\right)$ by

$$
\|\phi\|_{f}:=\sup \left\{\phi(\alpha)-\phi(\beta) \mid a_{\alpha} \neq 0, a_{\beta} \neq 0\right\} .
$$

Thus, to any square matrix $B$ over $\mathbb{E}\left[t_{1}^{ \pm 1}, \ldots, t_{m}^{ \pm 1}\right]$ with $\operatorname{det}(B) \neq 0$, we can associate a norm using $\operatorname{det}(B) \in \mathbb{F}\left[t_{1}^{ \pm 1}, \ldots, t_{m}^{ \pm 1}\right]$.

Generalizing this idea to the non-commutative case, in Section 2.1 we introduce the notion of a multivariable skew Laurent polynomial ring $\mathbb{K}\left[t_{1}^{ \pm 1}, \ldots, t_{m}^{ \pm 1}\right]$ of rank $m$ over a skew field $\mathbb{K}$. Given a square matrix $B$ over $\mathbb{K}\left[t_{1}^{ \pm 1}, \ldots, t_{m}^{ \pm 1}\right]$ we can study its Dieudonné determinant $\operatorname{det}(B)$ which is an element in the abelianization of the multiplicative group $\mathbb{K}\left(t_{1}, \ldots, t_{m}\right) \backslash\{0\}$ where $\mathbb{K}\left(t_{1}, \ldots, t_{m}\right)$ denotes the quotient field of $\mathbb{K}\left[t_{1}^{ \pm 1}, \ldots, t_{m}^{ \pm 1}\right]$. This determinant will in general not be represented by an element in $\mathbb{K}\left[t_{1}^{ \pm 1}, \ldots, t_{m}^{ \pm 1}\right]$. Our main technical result (Theorem 2.2) is that nonetheless there is a natural way to associate a norm to $B$ that generalizes the commutative case.

Algebraic 83 Geometric Topology, Volume 7 (2007) 
Given a 3-manifold $M$ and a 'compatible' representation

$$
\pi_{1}(M) \rightarrow \mathrm{GL}\left(\mathbb{K}\left[t_{1}^{ \pm 1}, \ldots, t_{m}^{ \pm 1}\right], d\right)
$$

we will show in Section 3 that the corresponding Reidemeister torsion can be viewed as a matrix over $\mathbb{K}\left[t_{1}^{ \pm 1}, \ldots, t_{m}^{ \pm 1}\right]$. Moreover, we will show in Section 4.3 that for appropriate representations the norm that we can associate to this matrix that agrees with $\rho_{n}, \delta_{n}$. This implies Theorem 1.1. We conclude this paper with examples of links for which we compute the Thurston norm using the results in this paper.

As a final remark we point out that the results in this paper completely generalize the results in [6]. Furthermore, the results can easily be extended to studying 2-complexes together with the Turaev norm which is modeled on the definition of the Thurston norm of a 3-manifold. We refer to Thurston [21] for details.

\section{Acknowledgments}

The authors would like to thank Tim Cochran, John Hempel, Taehee Kim and Chris Rasmussen for helpful conversations.

\section{The non-commutative Alexander norm}

In this section we will introduce the notion of a multivariable skew Laurent polynomial ring and we will then show that matrices over such rings give rise to semi-norms.

\subsection{Multivariable Laurent polynomials}

Let $\mathcal{R}$ be a (non-commutative) domain and $\gamma: \mathcal{R} \rightarrow \mathcal{R}$ a ring homomorphism. We denote by $\mathcal{R}\left[s^{ \pm 1}\right]$ the one-variable skew Laurent polynomial ring over $\mathcal{R}$. Specifically, the elements in $\mathcal{R}\left[s^{ \pm 1}\right]$ are formal sums $\sum_{i=m}^{n} a_{i} s^{i}(m \leq n \in \mathbb{Z})$ with $a_{i} \in \mathcal{R}$. Addition is given by addition of the coefficients, and multiplication is defined using the rule $s^{i} a=\gamma^{i}(a) s^{i}$ for any $a \in \mathcal{R}$ (where $\gamma^{i}(a)$ stands for $(\gamma \circ \cdots \circ \gamma)(a)$ ). We point out that any element $\sum_{i=m}^{n} a_{i} s^{i} \in \mathcal{R}\left[s^{ \pm 1}\right]$ can also be written uniquely in the form $\sum_{i=m}^{n} s^{i} \tilde{a}_{i}$, indeed, $\widetilde{a}_{i}=s^{-i} a_{i} s^{i} \in \mathcal{R}$.

In the following let $\mathbb{K}$ be a skew field. We then define a multivariable skew Laurent polynomial ring of rank $m$ over $\mathbb{K}$ (in non-commuting variables) to be a ring $R$ which is an algebra over $\mathbb{K}$ with unit (ie we can view $\mathbb{K}$ as a subring of $R$ ) together with a decomposition $R=\oplus_{\alpha \in \mathbb{Z}^{m}} V_{\alpha}$ such that the following hold:

(1) $V_{\alpha}$ is a one-dimensional $\mathbb{K}$-vector space, 
(2) $V_{\alpha} \cdot V_{\beta}=V_{\alpha+\beta}$ and

(3) $V_{(0, \ldots, 0)}=\mathbb{K}$.

In particular $R$ is $\mathbb{Z}^{m}$-graded. Note that these properties imply that any $V_{\alpha}$ is invariant under left and right multiplication by $\mathbb{K}$, that any element in $V_{\alpha} \backslash\{0\}$ is a unit, and that $R$ is a (non-commutative) domain. The example that the reader should keep in mind is a commutative Laurent polynomial ring $\mathbb{F}\left[t_{1}^{ \pm 1}, \ldots, t_{m}^{ \pm 1}\right]$. Indeed, let $t^{\alpha}:=t_{1}^{\alpha_{1}} \cdots \cdots t_{m}^{\alpha_{m}}$ for $\alpha=\left(\alpha_{1}, \ldots, \alpha_{m}\right)$, then $V_{\alpha}=\mathbb{F} t^{\alpha}, \alpha \in \mathbb{Z}^{m}$ has the required properties.

Let $R$ be a multivariable skew Laurent polynomial ring of rank $m$ over $\mathbb{K}$. To make our subsequent definitions and arguments easier to digest we will always pick $t^{\alpha} \in V_{\alpha} \backslash\{0\}$ for $\alpha \in \mathbb{Z}^{m}$. It is easy to see that we can in fact pick $t^{\alpha}, \alpha \in \mathbb{Z}^{m}$ such that $t^{n \alpha}=\left(t^{\alpha}\right)^{n}$ for all $\alpha \in \mathbb{Z}^{m}$ and $n \in \mathbb{Z}$. Note that this choice implies that $t^{(0, \ldots, 0)}=1$. Using the above choices, the set of $t^{\alpha}$ for $\alpha \in \mathbb{Z}$ satisfies the following properties:

(1) $t^{\alpha} t^{\tilde{\alpha}} t^{-(\alpha+\widetilde{\alpha})} \in \mathbb{K}^{\times}$for all $\alpha, \tilde{\alpha} \in \mathbb{Z}^{m}$ and

(2) $t^{\alpha} \mathbb{K}=\mathbb{K} t^{\alpha}$ for all $\alpha$.

This shows that the notion of multivariable skew Laurent polynomial ring of rank $m$ is a generalization of the notion of twisted group ring of $\mathbb{Z}^{m}$ as defined in Passman [13, page 13]. If $m=1$ then we have $t^{(n)} \in V_{(n)}$ such that $t^{(n)}=\left(t^{(1)}\right)^{n}$ for any $n \in \mathbb{Z}$. We write $t^{n}=t^{(n)}$. In particular, when $m=1, R$ is a one-variable skew Laurent polynomial ring as above.

The argument of Dodziuk et al [3, Corollary 6.3] can be used to show that any such Laurent polynomial ring is a (left and right) Ore domain and in particular has a (skew) quotient field. We normally denote a multivariable skew Laurent polynomial ring of

rank $m$ over $\mathbb{K}$ suggestively by $\mathbb{K}\left[t_{1}^{ \pm 1}, \ldots, t_{m}^{ \pm 1}\right]$ and we denote the quotient field of $\mathbb{K}\left[t_{1}^{ \pm 1}, \ldots, t_{m}^{ \pm 1}\right]$ by $\mathbb{K}\left(t_{1}, \ldots, t_{m}\right)$.

\subsection{The Dieudonné determinant}

In this section we recall several well-known definitions and facts about the Dieudonné determinant. Let $\mathcal{K}$ be a skew field; in our applications $\mathcal{K}$ will be the quotient field of a multivariable skew Laurent polynomial ring. First define $\operatorname{GL}(\mathcal{K}):=\lim \operatorname{GL}(\mathcal{K}, n)$, where we have the maps $\operatorname{GL}(\mathcal{K}, n) \rightarrow \operatorname{GL}(\mathcal{K}, n+1)$ in the direct system, given by $A \mapsto\left(\begin{array}{cc}A & 0 \\ 0 & 1\end{array}\right)$, then define $K_{1}(\mathcal{K})=\operatorname{GL}(\mathcal{K}) /[\operatorname{GL}(\mathcal{K}), \operatorname{GL}(\mathcal{K})]$. For details we refer the reader to Milnor [12] or Turaev [19].

Let $A$ a square matrix over $\mathcal{K}$. After elementary row operations and destabilization we can arrange that in $K_{1}(\mathcal{K})$ the matrix $A$ is represented by a $1 \times 1-$ matrix $(d)$. Then 
the Dieudonné determinant $\operatorname{det}(A) \in \mathcal{K}_{a b}^{\times}:=\mathcal{K}^{\times} /\left[\mathcal{K}^{\times}, \mathcal{K}^{\times}\right]\left(\right.$where $\left.\mathcal{K}^{\times}:=\mathcal{K} \backslash\{0\}\right)$ is defined to be $d$. It is well-known that the Dieudonné determinant induces an isomorphism det: $K_{1}(\mathcal{K}) \rightarrow \mathcal{K}_{a b}^{\times}$. We refer to Rosenberg [14, Theorem 2.2.5 and Corollary 2.2.6] for more details.

\subsection{Multivariable skew Laurent polynomial rings and semi-norms}

In this section we show that matrices defined over a multivariable skew Laurent polynomial ring give rise to a semi-norm. We also relate this norm to degrees of one-variable polynomials.

Let $\mathbb{K}\left[s^{ \pm 1}\right]$ be a one-variable skew Laurent polynomial ring and let $f \in \mathbb{K}\left[s^{ \pm 1}\right]$. If $f=$ 0 then we write $\operatorname{deg}(f)=-\infty$, otherwise, for $f=\sum_{i=m}^{n} a_{i} s^{i} \in \mathbb{K}\left[s^{ \pm 1}\right]$ with $a_{m} \neq$ $0, a_{n} \neq 0$ we define $\operatorname{deg}(f):=n-m$. This extends to a homomorphism deg: $\mathbb{K}(t) \backslash$ $\{0\} \rightarrow \mathbb{Z}$ via $\operatorname{deg}\left(f g^{-1}\right)=\operatorname{deg}(f)-\operatorname{deg}(g)$. Since $\operatorname{deg}$ is a homomorphism to an abelian group this induces a homomorphism deg: $\mathbb{K}(t)_{a b}^{\times} \rightarrow \mathbb{Z}$. Note that throughout this paper we will apply the convention that $-\infty<a$ for any $a \in \mathbb{Z}$.

For the remainder of this section let $\mathbb{K}\left[t_{1}^{ \pm 1}, \ldots, t_{m}^{ \pm 1}\right]$ be a multivariable skew Laurent polynomial ring of rank $m$ together with a choice of $t^{\alpha}, \alpha \in \mathbb{Z}^{m}$ as above. Let $f \in \mathbb{K}\left[t_{1}^{ \pm 1}, \ldots, t_{m}^{ \pm 1}\right]$. We can write $f=\sum_{\alpha \in \mathbb{Z}^{m}} a_{\alpha} t^{\alpha}$ for some $a_{\alpha} \in \mathbb{K}$. We associate a semi-norm $\|-\|_{f}$ on $\operatorname{hom}\left(\mathbb{R}^{m}, \mathbb{R}\right)$ to $f$ as follows. If $f=0$, then we set $\|-\|_{f}:=0$. Otherwise we set

$$
\|\phi\|_{f}:=\sup \left\{\phi(\alpha)-\phi(\beta) \mid a_{\alpha} \neq 0, a_{\beta} \neq 0\right\} .
$$

Clearly $\|-\|_{f}$ is a semi-norm and does not depend on the choice of $t^{\alpha}$. This semi-norm should be viewed as a generalization of the degree function.

Now let $\tau \in K_{1}\left(\mathbb{K}\left(t_{1}, \ldots, t_{m}\right)\right)$ and let $f_{n}, f_{d} \in \mathbb{K}\left[t_{1}^{ \pm 1}, \ldots, t_{m}^{ \pm 1}\right] \backslash\{0\}$ such that $\operatorname{det}(\tau)=f_{n} f_{d}^{-1} \in \mathbb{K}\left(t_{1}, \ldots, t_{m}\right)_{a b}^{\times}$. Then define

$$
\|\phi\|_{\tau}:=\max \left\{0,\|\phi\|_{f_{n}}-\|\phi\|_{f_{d}}\right\}
$$

for any $\phi \in \operatorname{hom}\left(\mathbb{R}^{m}, \mathbb{R}\right)$. By the following proposition this function is well-defined.

Proposition 2.1 Let $\tau \in K_{1}\left(\mathbb{K}\left(t_{1}, \ldots, t_{m}\right)\right)$. Let $f_{n}, f_{d}, g_{n}, g_{d} \in \mathbb{K}\left[t_{1}^{ \pm 1}, \ldots, t_{m}^{ \pm 1}\right] \backslash$ $\{0\}$ such that $\operatorname{det}(\tau)=f_{n} f_{d}^{-1}=g_{n} g_{d}^{-1} \in \mathbb{K}\left(t_{1}, \ldots, t_{m}\right)_{a b}^{\times}$. Then

$$
\|-\|_{f_{n}}-\|-\|_{f_{d}}=\|-\|_{g_{n}}-\|-\|_{g_{d}} \text {. }
$$

We postpone the proof to Section 2.4. 
Let $B$ be a matrix defined over $\mathbb{K}\left[t_{1}^{ \pm 1}, \ldots, t_{m}^{ \pm 1}\right]$. In general, it is not the case that $\operatorname{det}(B)$ can be represented by an element in $\mathbb{K}\left[t_{1}^{ \pm 1}, \ldots, t_{m}^{ \pm 1}\right]$. But we still have the following result which is the main technical result of this paper.

Theorem 2.2 If $\tau \in K_{1}\left(\mathbb{K}\left(t_{1}, \ldots, t_{m}\right)\right)$ can be represented by a matrix defined over $\mathbb{K}\left[t_{1}^{ \pm 1}, \ldots, t_{m}^{ \pm 1}\right]$, then $\|-\|_{\tau}$ defines a semi-norm on $\operatorname{hom}\left(\mathbb{R}^{m}, \mathbb{R}\right)$.

We postpone the proof to Section 2.5.

Now let $\phi: \mathbb{Z}^{m} \rightarrow \mathbb{Z}$ be a non-trivial homomorphism. We will show that $\|\phi\|_{B}$ can also be viewed as the degree of a polynomial associated to $B$ and $\phi$. We begin with some definitions. Consider

$$
\mathbb{K}[\operatorname{Ker}(\phi)]:=\bigoplus_{\alpha \in \operatorname{Ker}(\phi)} \mathbb{K} t^{\alpha} \subset \mathbb{K}\left[t_{1}^{ \pm 1}, \ldots, t_{m}^{ \pm 1}\right] .
$$

This clearly defines a subring of $\mathbb{K}\left[t_{1}^{ \pm 1}, \ldots, t_{m}^{ \pm 1}\right]$ and the argument of Dodziuk et al [3, Corollary 6.3] shows that $\mathbb{K}[\operatorname{Ker}(\phi)]$ is an Ore domain with skew field which we denote by $\mathbb{K}(\operatorname{Ker}(\phi))$.

Let $d \in \mathbb{Z}$ such that $\operatorname{Im}(\phi)=d \mathbb{Z}$ and pick $\beta=\left(\beta_{1}, \ldots, \beta_{m}\right) \in \mathbb{Z}^{m}$ such that $\phi(\beta)=d$. Let $\mu:=t^{\beta}$. Then we can form one-variable Laurent polynomial rings $(\mathbb{K}[\operatorname{Ker}(\phi)])\left[s^{ \pm 1}\right]$ and $\mathbb{K}(\operatorname{Ker}(\phi))\left[s^{ \pm 1}\right]$ where $s k:=\mu k \mu^{-1} s$ for all $k \in \mathbb{K}[\operatorname{Ker}(\phi)]$ respectively for all $k \in \mathbb{K}(\operatorname{Ker}(\phi))$. We get an isomorphism

$$
\begin{aligned}
\gamma_{\phi}: \mathbb{K}\left[t_{1}^{ \pm 1}, \ldots, t_{m}^{ \pm 1}\right] & \stackrel{\cong}{\rightrightarrows}(\mathbb{K}[\operatorname{Ker}(\phi)])\left[s^{ \pm 1}\right] \\
\sum_{\alpha \in \mathbb{Z}^{m}} k_{\alpha} t^{\alpha} & \mapsto \sum_{\alpha \in \mathbb{Z}^{m}} k_{\alpha} t^{\alpha} \mu^{-\phi(\alpha) / d_{S} \phi(\alpha) / d},
\end{aligned}
$$

where $k_{\alpha} \in \mathbb{K}$ for all $\alpha \in \mathbb{Z}^{m}$. Note that $k_{\alpha} t^{\alpha} \mu^{-\phi(\alpha) / d} \in \mathbb{K}[\operatorname{Ker}(\phi)]$. An easy computation shows that $\gamma_{\phi}$ is an isomorphism of rings. We also get an induced isomorphism $\mathbb{K}\left(t_{1}, \ldots, t_{m}\right) \stackrel{\cong}{\rightarrow}(\mathbb{K}(\operatorname{Ker}(\phi)))(s)$.

Let $B$ be a matrix over $\mathbb{K}\left(t_{1}, \ldots, t_{m}\right)$. Define $\operatorname{deg}_{\phi}(B):=\operatorname{deg}\left(\operatorname{det}\left(\gamma_{\phi}(B)\right)\right)$ where we view $\gamma(B)$ as a matrix over $\mathbb{K}(\operatorname{Ker}(\phi))(s)$.

Theorem 2.3 Let $B$ be a matrix over $\mathbb{R}\left(t_{1}, \ldots, t_{m}\right)$. Let $\phi \in \operatorname{hom}\left(\mathbb{Z}^{m}, \mathbb{Z}\right)$ be nontrivial and let $d \in \mathbb{N}$ such that $\operatorname{Im}(\phi)=d \mathbb{Z}$. Then

$$
\|\phi\|_{B}=d \max \left\{0, \operatorname{deg}_{\phi}(B)\right\} .
$$

In particular, this shows that $\operatorname{deg}_{\phi}(B)$ is independent of the choice of $\beta$. The above theorem is a generalization of [7, Proposition 5.12] to the non-commutative case. 
Proof Since $\gamma$ and deg are homomorphisms it is clearly enough to show that for any $g \in \mathbb{K}\left[t_{1}^{ \pm 1}, \ldots, t_{m}^{ \pm 1}\right] \backslash\{0\}$ we have

$$
\|\phi\|_{g}=d \operatorname{deg}\left(\gamma_{\phi}(g)\right) .
$$

Write $g=\sum_{\alpha \in \mathbb{Z}^{m}} a_{\alpha} t^{\alpha}$ with $a_{\alpha} \in \mathbb{K}$. Let $d, \beta, \mu$ and $\gamma: \mathbb{R}\left[t_{1}^{ \pm 1}, \ldots, t_{m}^{ \pm 1} \stackrel{\cong}{\rightarrow}\right.$ $(\mathbb{K}[\operatorname{Ker}(\phi)])\left[s^{ \pm 1}\right]$ as above. Note that $\operatorname{Ker}(\phi) \oplus \mathbb{Z} \beta=\mathbb{Z}^{m}$, hence

$$
\begin{aligned}
g & =\sum_{i \in \mathbb{Z}} \sum_{\alpha \in \operatorname{Ker}(\phi)} a_{\alpha+i \beta} t^{\alpha+i \beta}, \\
\gamma_{\phi}(g) & =\sum_{i \in \mathbb{Z}}\left(\sum_{\alpha \in \operatorname{Ker}(\phi)} a_{\alpha+i \beta} t^{\alpha+i \beta} \mu^{-i}\right) s^{i} .
\end{aligned}
$$

Note that $a_{\alpha+i \beta} t^{\alpha+i \beta} \mu^{-i} \subset \mathbb{K} t^{\alpha}$. Since $\mathbb{K}[\operatorname{Ker}(\phi)]=\oplus_{\alpha \in \operatorname{Ker}(\phi)} \mathbb{K} t^{\alpha}$ we get the following equivalences:

$$
\begin{aligned}
& \sum_{\alpha \in \operatorname{Ker}(\phi)} a_{\alpha+i \beta} t^{\alpha+i \beta} \mu^{-i}=0 \\
& \Leftrightarrow \quad a_{\alpha+i \beta} t^{\alpha+i \beta} \mu^{-i}=0 \text { for all } \alpha \in \operatorname{Ker}(\phi) \\
& \Leftrightarrow \quad a_{\alpha+i \beta}=0 \text { for all } \alpha \in \operatorname{Ker}(\phi) \text {. }
\end{aligned}
$$

Therefore

$$
\begin{aligned}
& \|\phi\|_{g}=\quad d \max _{i \in \mathbb{Z}}\left\{\text { there exists } \alpha \in \operatorname{Ker}(\phi) \text { such that } a_{\alpha+i \beta} \neq 0\right\} \\
& -d \min _{i \in \mathbb{Z}}\left\{\text { there exists } \alpha \in \operatorname{Ker}(\phi) \text { such that } a_{\alpha+i \beta} \neq 0\right\} \\
& =d \max _{i \in \mathbb{Z}}\left\{\sum_{\alpha \in \operatorname{Ker}(\phi)} a_{\alpha+i \beta} t^{\alpha+i \beta} \mu^{-i} \neq 0\right\} \\
& -d \min _{i \in \mathbb{Z}}\left\{\sum_{\alpha \in \operatorname{Ker}(\phi)} a_{\alpha+i \beta} t^{\alpha+i \beta} \mu^{-i} \neq 0\right\} \\
& =d \operatorname{deg}\left(\gamma_{\phi}(g)\right) \text {. }
\end{aligned}
$$

Thus the theorem is proved.

\subsection{Proof of Proposition 2.1}

We start out with the following three basic lemmas.

Lemma 2.4 Let $f, g \in \mathbb{K}\left[t_{1}^{ \pm 1}, \ldots, t_{m}^{ \pm 1}\right] \backslash\{0\}$, then $\|-\|_{f g}=\|-\|_{f}+\|-\|_{g}$.

This lemma is well-known. It follows from the fact that the Newton polytope of non-commutative multivariable polynomials $f g$ is the Minkowski sum of the Newton polytopes of $f$ and $g$. We refer to Sturmfels [16, page 31] for details.

Lemma 2.5 Let $d \in \mathbb{K}\left(t_{1}, \ldots, t_{m}\right)$ and let $f_{n}, f_{d}, g_{n}, g_{d} \in \mathbb{K}\left[t_{1}^{ \pm 1}, \ldots, t_{m}^{ \pm 1}\right]$ such that $d=f_{n} f_{d}^{-1}=g_{n} g_{d}^{-1} \in \mathbb{K}\left(t_{1}, \ldots, t_{m}\right)$. Then

$$
\|-\|_{f_{n}}-\|-\|_{f_{d}}=\|-\|_{g_{n}}-\|-\|_{g_{d}} .
$$

Algebraic $8 \mathcal{G}$ Geometric Topology, Volume 7 (2007) 
In particular

$$
\|-\|_{d}:=\|-\|_{f_{n}}-\|-\|_{f_{d}}
$$

is well-defined.

Proof Recall that by the definition of the Ore localization $f_{n} f_{d}^{-1}=g_{n} g_{d}^{-1} \in$ $\mathbb{K}\left(t_{1}, \ldots, t_{m}\right)$ is equivalent to the existence of $u, v \in \mathbb{K}\left[t_{1}^{ \pm 1}, \ldots, t_{m}^{ \pm 1}\right] \backslash\{0\}$ such that $f_{n} u=g_{n} v$ and $f_{d} u=g_{d} v$. The lemma now follows immediately from Lemma 2.4.

Lemma 2.6 Let $d, e \in \mathbb{K}\left(t_{1}, \ldots, t_{m}\right)$, then

$$
\|-\|_{d e}=\|-\|_{d}+\|-\|_{e} .
$$

Proof Pick $f_{n}, f_{d}, g_{n}, g_{d} \in \mathbb{K}\left[t_{1}^{ \pm 1}, \ldots, t_{m}^{ \pm 1}\right]$ such that $f_{n} f_{d}^{-1}=d$ and $g_{n} g_{d}^{-1}=e$. By the Ore property there exist $u, v \in \mathbb{K}\left[t_{1}^{ \pm 1}, \ldots, t_{m}^{ \pm 1}\right] \backslash\{0\}$ such that $g_{n} u=f_{d} v$. It follows that

$$
f_{n} f_{d}^{-1} g_{n} g_{d}^{-1}=f_{n} v u^{-1} g_{d}^{-1}=\left(f_{n} v\right)\left(g_{d} u\right)^{-1} .
$$

The lemma now follows immediately from Lemma 2.4.

We can now give the proof of Proposition 2.1.

Proof of Proposition 2.1 Let $B$ be a matrix defining an element $K_{1}\left(\mathbb{K}\left(t_{1}, \ldots, t_{m}\right)\right)$. Assume that we have $f_{n}, f_{d}, g_{n}, g_{d} \in \mathbb{K}\left[t_{1}^{ \pm 1}, \ldots, t_{m}^{ \pm 1}\right]$ such that $\operatorname{det}(B)=f_{n} f_{d}^{-1}=$ $g_{n} g_{d}^{-1} \in \mathbb{K}\left(t_{1}, \ldots, t_{m}\right)_{a b}^{\times}$. We can lift the equality $f_{n} f_{d}^{-1}=g_{n} g_{d}^{-1} \in \mathbb{K}\left(t_{1}, \ldots, t_{m}\right)_{a b}^{\times}$ to an equality

$$
f_{n} f_{d}^{-1}=\prod_{i=1}^{r}\left[a_{i}, b_{i}\right] g_{n} g_{d}^{-1} \in \mathbb{K}\left(t_{1}, \ldots, t_{m}\right)^{\times}
$$

for some $a_{i}, b_{i} \in \mathbb{K}\left(t_{1}, \ldots, t_{m}\right)$. It follows from Lemma 2.6 that $\|-\|_{\left[a_{i}, b_{i}\right]}=0$. It then follows from Lemma 2.6 that $\|-\|_{f_{n} f_{d}^{-1}}=\|-\|_{g_{n} g_{d}^{-1}}$.

\subsection{Proof of Theorem 2.2}

Let $\tau \in K_{1}\left(\mathbb{K}\left(t_{1}, \ldots, t_{m}\right)\right)$ that can be represented by a matrix $B$ defined over $\mathbb{K}\left[t_{1}^{ \pm 1}, \ldots, t_{m}^{ \pm 1}\right]$. We will show that $\|-\|_{\tau}=\|-\|_{B}$ defines a semi-norm on hom $\left(\mathbb{R}^{m}, \mathbb{R}\right)$. Because of the continuity and the $\mathbb{N}$-linearity of $\|-\|_{B}$ it is enough to show that for any two non-trivial homomorphisms $\phi, \tilde{\phi}: \mathbb{Z}^{m} \rightarrow \mathbb{Z}$ we have

$$
\|\phi+\tilde{\phi}\|_{B} \leq\|\phi\|_{B}+\|\tilde{\phi}\|_{B} .
$$

Algebraic 83 Geometric Topology, Volume 7 (2007) 
Let $\phi, \tilde{\phi}: \mathbb{Z}^{m} \rightarrow \mathbb{Z}$ be non-trivial homomorphisms. Let $d \in \mathbb{Z}$ such that $\operatorname{Im}(\phi)=d \mathbb{Z}$ and pick $\beta$ with $\phi(\beta)=d$. We write $\mu=t^{\beta}$. As in Section 2.3 we can form $\mathbb{K}[\operatorname{Ker}(\phi)]$ and we also have an isomorphism $\gamma_{\phi}: \mathbb{K}\left[t_{1}^{ \pm 1}, \ldots, t_{m}^{ \pm 1}\right] \stackrel{\cong}{\rightarrow}(\mathbb{K}[\operatorname{Ker}(\phi)])\left[s^{ \pm 1}\right]$.

Consider $\gamma_{\phi}(B)$, it is defined over the PID $\mathbb{K}(\operatorname{Ker}(\phi))\left[s^{ \pm 1}\right]$. Therefore we can use elementary row operations to turn $\gamma_{\phi}(B)$ into a diagonal matrix with entries in $\mathbb{K}(\operatorname{Ker}(\phi))\left[s^{ \pm 1}\right]$. In particular we can find $a_{i}, b_{i} \in \mathbb{K}[\operatorname{Ker}(\phi)]$ such that

$$
\operatorname{det}\left(\gamma_{\phi}(B)\right)=\sum_{i=r_{1}}^{r_{2}} s^{i} a_{i} b_{i}^{-1}
$$

Since $\mathbb{K}[\operatorname{Ker}(\phi)]$ is an Ore domain we can in fact find a common denominator for $a_{i} b_{i}^{-1}, i=r_{1}, \ldots, r_{2}$. More precisely, we can find $c_{r_{1}}, \ldots, c_{r_{2}} \in \mathbb{K}[\operatorname{Ker}(\phi)]$ and $d \in \mathbb{K}[\operatorname{Ker}(\phi)]$ such that $a_{i} b_{i}^{-1}=c_{i} d^{-1}$ for $i=r_{1}, \ldots, r_{2}$. Now let $c=\sum_{i=r_{1}}^{r_{2}} s^{i} c_{i}$. Then

$$
\operatorname{det}\left(\gamma_{\phi}(B)\right)=c d^{-1} \in \mathbb{K}(\operatorname{Ker}(\phi))(s)_{a b}^{\times}
$$

where $c \in \mathbb{K}[\operatorname{Ker}(\phi)]\left[s^{ \pm 1}\right]$ and $d \in \mathbb{K}[\operatorname{Ker}(\phi)]$. Now let $f=\gamma_{\phi}^{-1}(c) \in \mathbb{K}\left[t_{1}^{ \pm 1}, \ldots, t_{m}^{ \pm 1}\right]$ and $g=\gamma_{\phi}^{-1}(d) \in \mathbb{K}[\operatorname{Ker}(\phi)]$. Then $\operatorname{det}(B)=f g^{-1}$ and by Proposition 2.1 we have

$$
\|-\|_{B}=\|-\|_{f}-\|-\|_{g}
$$

We now observe that $\|\phi\|_{g}=0$ and $\|\phi+\tilde{\phi}\|_{g}=\|\tilde{\phi}\|_{g}$ since $g \in \mathbb{K}[\operatorname{Ker}(\phi)]$. Therefore it follows that

$$
\begin{aligned}
\|\phi+\tilde{\phi}\|_{B} & =\|\phi+\tilde{\phi}\|_{f}-\|\phi+\tilde{\phi}\|_{g} \\
& =\|\phi+\widetilde{\phi}\|_{f}-\|\tilde{\phi}\|_{g} \\
& \leq\|\phi\|_{f}+\|\tilde{\phi}\|_{f}-\|\tilde{\phi}\|_{g} \\
& =\left(\|\phi\|_{f}-\|\phi\|_{g}\right)+\left(\|\tilde{\phi}\|_{f}-\|\tilde{\phi}\|_{g}\right) \\
& =\|\phi\|_{B}+\|\tilde{\phi}\|_{B} .
\end{aligned}
$$

This concludes the proof of Theorem 2.2.

\section{Applications to the Thurston norm}

In this section we will show that the Reidemeister torsion corresponding to 'compatible' representations over a multivariable skew Laurent polynomial ring give rise to seminorms that give lower bounds on the Thurston norm.

Algebraic $8 \mathcal{G}$ Geometric Topology, Volume 7 (2007) 


\subsection{Reidemeister torsion}

Let $X$ be a finite connected CW-complex. Denote the universal cover of $X$ by $\tilde{X}$. We view $C_{*}(\tilde{X})$ as a right $\mathbb{Z}\left[\pi_{1}(X)\right]$-module via deck transformations. Let $R$ be a ring and let $\varphi: \pi_{1}(X) \rightarrow \operatorname{GL}(R, d)$ be a representation. This equips $R^{d}$ with a left $\mathbb{Z}\left[\pi_{1}(X)\right]$-module structure. We can therefore consider the right $R$-module chain complex $C_{*}^{\varphi}\left(X ; R^{d}\right):=C_{*}(\tilde{X}) \otimes_{\mathbb{Z}\left[\pi_{1}(X)\right]} R^{d}$. We denote its homology by $H_{i}^{\varphi}\left(X ; R^{d}\right)$. If $H_{*}^{\varphi}\left(X ; R^{d}\right)=0$, then we define the Reidemeister torsion $\tau(X, \varphi) \in$ $K_{1}(R) / \pm \varphi\left(\pi_{1}(X)\right)$ otherwise we write $\tau(X, \varphi):=0$. If the homomorphism $\varphi$ is clear we may also write $\tau\left(X, R^{d}\right)$.

Let $M$ be a manifold. Since Reidemeister torsion only depends on the homeomorphism type of the space we can define $\tau(M, \varphi)$ by picking any CW-structure for $M$. We refer to the excellent book of Turaev [19] for the details.

\subsection{Compatible homomorphisms and the higher order Alexander norm}

In the following let $M$ be a 3-manifold with empty or toroidal boundary. Let $\psi: H_{1}(M) \rightarrow \mathbb{Z}^{m}$ be an epimorphism. Let $\mathbb{K}\left[t_{1}^{ \pm 1}, \ldots, t_{m}^{ \pm 1}\right]$ be a multivariable skew Laurent polynomial ring of rank $m$ as in Section 2.1.

A representation $\varphi: \pi_{1}(M) \rightarrow \mathrm{GL}\left(\mathbb{K}\left[t_{1}^{ \pm 1}, \ldots, t_{m}^{ \pm 1}\right], d\right)$ is called $\psi$-compatible if for any $g \in \pi_{1}(X)$ we have $\varphi(g)=A t^{\psi(g)}$ for some $A \in \mathrm{GL}(\mathbb{K}, d)$. This generalizes definitions in Turaev [20] and Friedl [4]. We denote the induced representation $\pi_{1}(M) \rightarrow \operatorname{GL}\left(\mathbb{K}\left(t_{1}, \ldots, t_{m}\right), d\right)$ by $\varphi$ as well and consider the corresponding Reidemeister torsion $\tau(M, \varphi) \in K_{1}\left(\mathbb{K}\left(t_{1}, \ldots, t_{m}\right)\right) / \pm \varphi\left(\pi_{1}(M)\right) \cup\{0\}$.

We say $\varphi$ is a commutative representation if there exists a commutative subfield $\mathbb{F}$ of $\mathbb{K}$ such that for all $g$ we have $\varphi(g)=A t^{\psi(g)}$ with $A$ defined over $\mathbb{F}$ and if $t^{\alpha}, t^{\tilde{\alpha}}$ commute for any $\alpha, \tilde{\alpha} \in \mathbb{Z}^{m}$. The following result is our main application of the purely algebraic results of Section 2.

Theorem 3.1 Let $M$ be a 3-manifold with an empty or toroidal boundary, let $\psi: H_{1}(M) \rightarrow \mathbb{Z}^{m}$ be an epimorphism and let $\varphi: \pi_{1}(M) \rightarrow G L\left(\mathbb{K}\left[t_{1}^{ \pm 1}, \ldots, t_{m}^{ \pm 1}\right], d\right)$ be a $\psi$-compatible representation such that $\tau(M, \varphi) \neq 0$. If one of the following holds:

(1) $\varphi$ is commutative or

(2) there exists $g \in \operatorname{Ker}\left\{\pi_{1}(M) \rightarrow \mathbb{Z}^{m}\right\}$ such that $\varphi(g)-$ id is invertible over $\mathbb{K}$, then $\|-\|_{\tau(M, \varphi)}$ is a semi-norm on hom $\left(\mathbb{R}^{m}, \mathbb{R}\right)$ and for any $\phi: \mathbb{R}^{m} \rightarrow \mathbb{R}$ we have

$$
\|\phi \circ \psi\|_{T} \geq\|\phi\|_{\tau(M, \varphi)} .
$$


We point out that if $g \in \operatorname{Ker}\left\{\pi_{1}(M) \rightarrow \mathbb{Z}^{m}\right\}$, then $\varphi(g)-\mathrm{id}$ is defined over $\mathbb{K}$ since $\varphi$ is $\psi$-compatible. We refer to $\|-\|_{\tau(M, \varphi)}$ as the higher-order Alexander norm.

In the case that $\mathbb{K}\left[t_{1}^{ \pm 1}, \ldots, t_{m}^{ \pm 1}\right]$ equals $\mathbb{Q}\left[t_{1}^{ \pm 1}, \ldots, t_{m}^{ \pm 1}\right]$, the usual commutative Laurent polynomial ring, we recover McMullen's Alexander norm $\|-\|_{A}$ (cf McMullen [11]). The general commutative case is the main result in Friedl-Kim [6]. The proof we give here is different in its nature from the proofs in [11] and [6].

Proof In the case that $m=1$ it is clear that $\|-\|_{\tau(M, \varphi)}$ is a semi-norm. The fact that it gives a lower bound on the Thurston norm was shown in $[1 ; 7 ; 20 ; 4]$. We therefore assume now that $m>1$.

We first show that $\|\phi \circ \psi\|_{T} \geq\|\phi\|_{\tau(M, \varphi)}$ for any $\phi: \mathbb{R}^{m} \rightarrow \mathbb{R}$. Since both sides are $\mathbb{N}$-linear and continuous we only have to show that $\|\phi \circ \psi\|_{T} \geq\|\phi\|_{\tau(M, \varphi)}$ for all epimorphisms $\phi: \mathbb{Z}^{m} \rightarrow \mathbb{Z}$. So from now on, we will assume that $\phi: \mathbb{Z}^{m} \rightarrow \mathbb{Z}$ is an epimorphism.

Pick $\mu \in \mathbb{Z}^{m}$ with $\phi(\mu)=1$ as in the definition of $\operatorname{deg}_{\phi}(\tau(M, \varphi))$. We can then form the rings $\mathbb{K}[\operatorname{Ker}(\phi)]\left[s^{ \pm 1}\right]$ and $\mathbb{K}(\operatorname{Ker}(\phi))(s)$. First note that by Theorem 2.3

$$
\|\phi\|_{\tau(M, \varphi)}=\operatorname{deg}_{\phi}(\tau(M, \varphi))
$$

since $\phi$ is surjective. The representation

$$
\pi_{1}(M) \rightarrow \operatorname{GL}\left(\mathbb{K}\left[t_{1}^{ \pm 1}, \ldots, t_{m}^{ \pm 1}\right], d\right) \rightarrow \operatorname{GL}\left(\mathbb{K}(\operatorname{Ker}(\phi))\left[s^{ \pm 1}\right], d\right)
$$

is $\phi$-compatible since $\pi_{1}(M) \rightarrow \mathrm{GL}\left(\mathbb{K}\left[t_{1}^{ \pm 1}, \ldots, t_{m}^{ \pm 1}\right], d\right)$ is $\psi$-compatible. It now follows from Friedl [4, Theorem 1.2] that $\|\phi \circ \psi\|_{T} \geq \operatorname{deg}(\tau(M, \mathbb{K}(\operatorname{Ker}(\phi))(s)))=$ $\operatorname{deg}_{\phi}(\tau(M, \varphi))$ (cf also Turaev [20]).

In the remainder of the proof we will show that if $m>1$ then the Reidemeister torsion $\tau(M, \varphi) \in K_{1}\left(\mathbb{K}\left(t_{1}, \ldots, t_{m}\right)\right) / \pm \varphi\left(\pi_{1}(M)\right)$ can be represented by a matrix defined over $\mathbb{K}\left[t_{1}^{ \pm 1}, \ldots, t_{m}^{ \pm 1}\right]$. It then follows from Theorem 2.2 that $\|-\|_{\tau(M, \varphi)}$ is a semi-norm.

Consider the case that $\varphi$ is a commutative representation and let $\mathbb{F}$ be the commutative subfield $\mathbb{F}$ in the definition of a commutative representation. Denote by $\mathbb{F}\left[t_{1}^{ \pm 1}, \ldots, t_{m}^{ \pm 1}\right]$ the ordinary Laurent polynomial ring. Then we have $\psi$-compatible representations $\pi_{1}(M) \rightarrow \mathrm{GL}\left(\mathbb{E}\left[t_{1}^{ \pm 1}, \ldots, t_{m}^{ \pm 1}\right], d\right) \hookrightarrow \mathrm{GL}\left(\mathbb{K}\left[t_{1}^{ \pm 1}, \ldots, t_{m}^{ \pm 1}\right], d\right)$. By [19, Proposition 3.6] we have

$$
\tau\left(M, \mathbb{F}\left(t_{1}, \ldots, t_{m}\right)\right)=\tau\left(M, \mathbb{K}\left(t_{1}, \ldots, t_{m}\right)\right) \in K_{1}\left(\mathbb{K}\left(t_{1}, \ldots, t_{m}\right)\right) / \pm \varphi\left(\pi_{1}(M)\right) .
$$

Since $m>1$ it follows from [19, Theorem 4.7] combined with [6, Lemmas 6.2 and 6.5] that $\operatorname{det}\left(\tau\left(M, \mathbb{F}\left(t_{1}, \ldots, t_{m}\right)\right)\right) \in \mathbb{F}\left(t_{1}, \ldots, t_{m}\right)$ equals the twisted multivariable 
Alexander polynomial, in particular it is defined over $\mathbb{E}\left[t_{1}^{ \pm 1}, \ldots, t_{m}^{ \pm 1}\right]$. This concludes the proof in the commutative case.

It therefore remains to consider the case when there exists $g \in \operatorname{Ker}\left\{\pi_{1}(M) \rightarrow \mathbb{Z}^{m}\right\}$ such that $\varphi(g)-$ id is invertible. We first consider the case that $M$ is a closed 3-manifold. The proof will use the special CW-structure from the next claim.

Claim There exists a CW-structure for $M$ with one $0-$ cell and one 3-cell and such the closure of a 1-cell and the cocore of a 2-cell represent $g .{ }^{1}$

In order to prove the claim pick a Heegaard decomposition $M=G_{0} \cup H_{0}$. We can add a handle $g_{0}$ (respectively $h_{0}$ ) to $G_{0}$ (respectively $H_{0}$ ) in $H_{0}$ (respectively $G_{0}$ ) so that the core of $g_{0}$ (respectively $h_{0}$ ) represents $g$. Adding further handles $h_{1}, \ldots, h_{r}$ (respectively $\left.g_{1}, \ldots, g_{r}\right)$ in $M \backslash G_{0}$ (respectively $M \backslash H_{0}$ ) we can assume that complement $H_{0} \backslash\left(\bigcup_{i=0}^{r} g_{i}\right)$ (respectively $G_{0} \backslash\left(\bigcup_{i=0}^{s} h_{i}\right)$ ) is again a handlebody. It follows that $G:=\left(G_{0} \cup \bigcup_{i=0}^{r} g_{i}\right) \backslash\left(\bigcup_{i=0}^{s} h_{i}\right)$ and $H:=\left(H_{0} \cup \bigcup_{i=0}^{s} h_{i}\right) \backslash\left(\bigcup_{i=0}^{r} g_{i}\right)$ are handlebodies and hence $M=G \cup H$ is a handlebody decomposition of $M$.

Now give $M$ the CW structure as follows: take one 0-cell, attach 1-cells along a choice of cores of $G$ such that $g$ is represented by the closure of a 1-cell. Attach 2-cells along cocores of $H$ such that one cocore represents $g$. Finally attach one 3-cell. This CW-structure clearly has the required properties to complete the claim.

Denote the number of 1-cells by $n$. Consider the chain complex of the universal cover $\tilde{M}$ :

$$
0 \rightarrow C_{3}(\tilde{M})^{1} \stackrel{\partial_{3}}{\longrightarrow} C_{2}(\tilde{M})^{n} \stackrel{\partial_{2}}{\longrightarrow} C_{1}(\tilde{M})^{n} \stackrel{\partial_{1}}{\longrightarrow} C_{0}(\tilde{M})^{1} \rightarrow 0,
$$

where the superscript indicates the rank over $\mathbb{Z}\left[\pi_{1}(M)\right]$. Picking appropriate lifts of the cells of $M$ to cells of $\tilde{M}$ and picking an appropriate order we get bases for the $\mathbb{Z}\left[\pi_{1}(M)\right]$-modules $C_{i}(\tilde{M})$, such that if $A_{i}$ denotes the matrix corresponding to $\partial_{i}$, then $A_{1}$ and $A_{3}$ are of the form

$$
\begin{aligned}
& A_{3}=\left(1-g, 1-b_{1}, \ldots, 1-b_{n-1}\right)^{t}, \\
& A_{1}=\left(1-g, 1-a_{1}, \ldots, 1-a_{n-1}\right),
\end{aligned}
$$

for some $a_{i}, b_{i} \in \pi_{1}(M), i=1, \ldots, n-1$. By assumption id $-\varphi(g)$ is invertible over $\mathbb{K}$. Denote by $B_{2}$ the result of deleting the first column and the first row of $A_{2}$. Let $\tau:=(\mathrm{id}-\varphi(g))^{-1} \varphi\left(B_{2}\right)(\mathrm{id}-\varphi(g))^{-1}$. Note that $\tau$ is defined over $\mathbb{K}\left[t_{1}^{ \pm 1}, \ldots, t_{m}^{ \pm 1}\right]$. Since we assume that $\tau(M, \varphi) \neq 0$ it follows that $\varphi\left(B_{2}\right)$ is invertible over $\mathbb{K}\left(t_{1}, \ldots, t_{m}\right)$ and $\tau(M, \varphi)=\tau \in K_{1}\left(\mathbb{K}\left(t_{1}, \ldots, t_{m}\right)\right) / \pm \varphi\left(\pi_{1}(M)\right)$ (we refer to [19, Theorem 2.2]

\footnotetext{
${ }^{1}$ By cocore, we mean the element in $\pi_{1}$ given by taking a point on the $2-$ cell and connecting the two push-offs through an arc in the 3-cell.
} 
for details). Therefore $\tau(M, \varphi) \in K_{1}\left(\mathbb{K}\left(t_{1}, \ldots, t_{m}\right)\right) / \pm \varphi\left(\pi_{1}(M)\right)$ can be represented by a matrix defined over $\mathbb{K}\left[t_{1}^{ \pm 1}, \ldots, t_{m}^{ \pm 1}\right]$. This concludes the proof in the case that $M$ is a closed 3-manifold.

In the case that $M$ is a 3-manifold with non-empty toroidal boundary we can find a (simple) homotopy equivalence to a 2-complex $X$ with $\chi(X)=0$. We can assume that the CW-structure has one 0 -cell, $n$ 1-cells and $n-12$-cells, furthermore we can assume that the closure of a $1-$ cell represents an element $g \in \operatorname{Ker}\left\{\psi: \pi_{1}(X) \rightarrow \mathbb{Z}^{m}\right\}$ such that id $-\varphi(g)$ is invertible. We get a chain complex

$$
0 \rightarrow C_{2}(\tilde{X})^{n-1} \stackrel{\partial_{2}}{\rightarrow} C_{1}(\tilde{X})^{n} \stackrel{\partial_{1}}{\rightarrow} C_{0}(\tilde{X})^{1} \rightarrow 0 .
$$

Picking appropriate lifts of the cells of $X$ to cells of $\tilde{X}$ we get bases for the $\mathbb{Z}\left[\pi_{1}(X)\right]-$ modules $C_{i}(\tilde{X})$, such that if $A_{i}$ denotes the matrix corresponding to $\partial_{i}$, then $A_{1}$ is of the form

$$
A_{1}=\left(1-g, 1-a_{1}, \ldots, 1-a_{n-1}\right),
$$

for some $a_{i} \in \pi_{1}(M)$. Now denote by $B_{2}$ the result of deleting the first row of $A_{2}$. Then $\tau:=\varphi\left(B_{2}\right)(\mathrm{id}-\varphi(g))^{-1}$ is again defined over $\mathbb{K}\left[t_{1}^{ \pm 1}, \ldots, t_{m}^{ \pm 1}\right]$ and the proof continues as in the case of a closed 3-manifold.

Remark It follows from [4] that if $M$ is closed, or if $M$ has toroidal boundary, then $\tau(M, \varphi) \neq 0$ is equivalent to $H_{1}\left(M ; \mathbb{K}\left(t_{1}, \ldots, t_{m}\right)\right)=0$, or equivalently, that $H_{1}\left(M ; \mathbb{K}\left[t_{1}^{ \pm 1}, \ldots, t_{m}^{ \pm 1}\right]\right)$ has rank zero over $\mathbb{K}\left[t_{1}^{ \pm 1}, \ldots, t_{m}^{ \pm 1}\right]$.

Remark The computation of polynomials

$$
f_{d} \in \mathbb{K}\left[t_{1}^{ \pm 1}, \ldots, t_{m}^{ \pm 1}\right] \text { and } f_{n} \in \mathbb{K}\left[t_{1}^{ \pm 1}, \ldots, t_{m}^{ \pm 1}\right]
$$

such that $\operatorname{det}(\tau(M, \varphi))=f_{n} f_{d}^{-1}$ is computationally equivalent to the computation of $\operatorname{deg}_{\phi}(\tau(M, \varphi))$ for some $\phi: H_{1}(M) \rightarrow \mathbb{Z}$. Put differently we get the perhaps surprising fact that computing the higher-order Alexander norm does not take longer than computing a single higher-order, one-variable Alexander polynomial.

\section{Examples of $\psi$-compatible homomorphisms}

Following [1] and [7] we will use the rational derived series to give examples of $\psi-$ compatible homomorphisms. For a given a 3-manifold, we will show that these give rise to a never decreasing, eventually constant sequence of semi-norms all of which give lower bounds on the Thurston norm.

Algebraic $\mathcal{E} \mathcal{G}$ Geometric Topology, Volume 7 (2007) 


\subsection{Skew fields of group rings}

A group $G$ is called locally indicable if for every finitely generated non-trivial subgroup $U \subset G$ there exists a non-trivial homomorphism $U \rightarrow \mathbb{Z}$. We recall the following well-known theorem.

Theorem 4.1 Let $G$ be a locally indicable and amenable group and let $R$ be a subring of $\mathbb{C}$. Then $R[G]$ is an Ore domain, in particular it embeds in its classical right ring of quotients $\mathbb{K}(G)$.

Higman [9] showed that $R[G]$ has no zero divisors. The theorem now follows from [17] or [3, Corollary 6.3].

We recall that a group $G$ is called poly-torsion-free-abelian (PTFA) if there exists a filtration

$$
1=G_{0} \subset G_{1} \subset \cdots \subset G_{n-1} \subset G_{n}=G
$$

such that $G_{i} / G_{i-1}$ is torsion free abelian. It is well-known that PTFA groups are amenable and locally indicable (cf [15]). The group rings of PTFA groups played an important role in Cochran-Orr-Teichner [2], Cochran [1] and Harvey [7].

\subsection{Admissible pairs and multivariable skew Laurent polynomial rings}

We slightly generalize a definition from Harvey [8].

Definition Let $\pi$ be a group and let $\psi: \pi \rightarrow \mathbb{Z}^{m}$ be an epimorphism and let $\varphi: \pi \rightarrow G$ be an epimorphism to a locally indicable and amenable group $G$ such that there exists a map $G \rightarrow \mathbb{Z}^{m}$ (which we also denote by $\psi$ ) such that

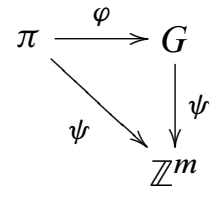

commutes. Following [8, Definition 1.4] we call $(\varphi, \psi)$ an admissible pair for $\pi$.

Clearly $G_{\psi}:=\operatorname{Ker}\left\{G \rightarrow \mathbb{Z}^{m}\right\}$ is locally indicable and amenable. It follows now from Passman [13, Lemma 3.5 (ii)] that $\left(\mathbb{Z}[G], \mathbb{Z}\left[G_{\psi}\right] \backslash\{0\}\right)$ satisfies the Ore property. Now pick elements $t^{\alpha} \in G, \alpha \in \mathbb{Z}^{m}$ such that $\psi\left(t^{\alpha}\right)=\alpha$ and $t^{n \alpha}=\left(t^{\alpha}\right)^{n}$ for any $\alpha \in \mathbb{Z}^{m}, n \in \mathbb{Z}$.

Clearly $\mathbb{Z}[G]\left(\mathbb{Z}\left[G_{\psi}\right] \backslash\{0\}\right)^{-1}=\sum_{\alpha \in \mathbb{Z}^{m}} \mathbb{K}\left(G_{\psi}\right) t^{\alpha}$ is a multivariable skew Laurent polynomial ring of rank $m$ over the field $\mathbb{K}\left(G_{\psi}\right)$ as defined in Section 2.1. We denote 
this ring by $\mathbb{K}\left(G_{\psi}\right)\left[t_{1}^{ \pm 1}, \ldots, t_{m}^{ \pm 1}\right]$. Note that $\mathbb{Z}[\pi] \rightarrow \mathbb{Z}[G] \rightarrow \mathbb{K}\left(G_{\psi}\right)\left[t_{1}^{ \pm 1}, \ldots, t_{m}^{ \pm 1}\right]$ is a $\psi$-compatible homomorphism and that $\mathbb{K}\left(G_{\psi}\right)\left(t_{1}, \ldots, t_{m}\right)$ is canonically isomorphic to $\mathbb{K}(G)$.

A family of examples of admissible pairs is provided by the rational derived series of a group $\pi$ introduced by the second author (cf [7, Section 3]). Let $\pi_{r}^{(0)}:=\pi$ and define inductively

$$
\pi_{r}^{(n)}:=\left\{g \in \pi_{r}^{(n-1)} \mid g^{d} \in\left[\pi_{r}^{(n-1)}, \pi_{r}^{(n-1)}\right] \text { for some } d \in \mathbb{Z} \backslash\{0\}\right\} .
$$

Note that $\pi_{r}^{(n-1)} / \pi_{r}^{(n)} \cong\left(\pi_{r}^{(n-1)} /\left[\pi_{r}^{(n-1)}, \pi_{r}^{(n-1)}\right]\right) / \mathbb{Z}$-torsion. By [7, Corollary 3.6] the quotients $\pi / \pi_{r}^{(n)}$ are PTFA groups for any $\pi$ and any $n$. If $\psi: \pi \rightarrow \mathbb{Z}^{m}$ is an epimorphism, then $\left(\pi \rightarrow \pi / \pi_{r}^{(n)}, \psi\right)$ is an admissible pair for $\pi$ for any $n>0$.

\subsection{Admissible pairs and semi-norms}

Let $M$ be a 3-manifold with empty or toroidal boundary. Let

$$
\left(\varphi: \pi_{1}(M) \rightarrow G, \psi: \pi_{1}(M) \rightarrow \mathbb{Z}^{m}\right)
$$

be an admissible pair for $\pi_{1}(M)$. We denote the induced map

$$
\mathbb{Z}\left[\pi_{1}(M)\right] \rightarrow \mathbb{K}\left(G_{\psi}\right)\left(t_{1}, \ldots, t_{m}\right)
$$

by $\varphi$ as well.

Let $\phi: \mathbb{Z}^{m} \rightarrow \mathbb{Z}$ be a non-trivial homomorphism. We denote the induced homomorphism $G \rightarrow \mathbb{Z}^{m} \rightarrow \mathbb{Z}$ by $\phi$ as well. We write $G_{\phi}:=\operatorname{Ker}\{G \rightarrow \mathbb{Z}\}$. Pick $\mu \in G$ such that $\phi(\mu) \mathbb{Z}=\operatorname{Im}(\phi)$. We define $\mathbb{Z}\left[G_{\phi}\right]\left[u^{ \pm 1}\right]$ via $u f=\mu f \mu^{-1} u$. Note that we get an isomorphism $\mathbb{K}\left(G_{\phi}\right)(u) \cong \mathbb{K}(G)$. If $\tau(M, \varphi) \neq 0$, then we define

$$
\delta_{G}(\phi):=\max \left\{0, \operatorname{deg}\left(\tau\left(M, \mathbb{K}\left(G_{\phi}\right)(u)\right)\right)\right\},
$$

otherwise we write $\delta_{G}(\phi)=-\infty$. We will adopt the convention that $-\infty<a$ for any $a \in \mathbb{Z}$. By [4] this agrees with the definition in [8, Definition 1.6] if $\delta_{G}(\phi) \neq-\infty$ and if $\varphi: G \rightarrow \mathbb{Z}^{m}$ is not an isomorphism or if $m>1$. In the case that $\varphi: G \rightarrow \mathbb{Z}$ is an isomorphism and $M \neq S^{1} \times D^{2}, S^{1} \times S^{2}$, this definition differs from [8, Definition 1.6] by the term $1+b_{3}(M)$. In the case that $\varphi: \pi \rightarrow \pi / \pi_{r}^{(n+1)}$ then we also write $\delta_{n}(\phi)=\delta_{\pi / \pi_{r}^{(n+1)}}(\phi)$.

The following theorem implies Theorem 1.1.

Theorem 4.2 Let $M$ be a 3-manifold with an empty or toroidal boundary. Let $\left(\varphi: \pi_{1}(M) \rightarrow G, \psi: \pi_{1}(M) \rightarrow \mathbb{Z}^{m}\right)$ be an admissible pair for $\pi_{1}(M)$ such that 
$\tau(M, \varphi) \neq 0$. Then for any $\phi: \mathbb{Z}^{m} \rightarrow \mathbb{Z}$ we have $\|\phi\|_{\tau(M, \varphi)}=\delta_{G}(\phi)$ and $\phi \mapsto$ $\max \left\{0, \delta_{G}(\phi)\right\}$ defines a semi-norm which is a lower bound on the Thurston norm.

Proof Let $\phi: \mathbb{Z}^{m} \rightarrow \mathbb{Z}$ be a non-trivial homomorphism. As in Section 2.1 we can form $\mathbb{K}\left(G_{\phi}\right)\left[s^{ \pm 1}\right]$ and $\mathbb{K}\left(G_{\psi}\right)(\operatorname{Ker}(\phi))\left[s^{ \pm 1}\right]$. Note that these rings are canonically isomorphic Laurent polynomial rings. If $\psi: G \rightarrow \mathbb{Z}^{m}$ is an isomorphism, then $\varphi$ is commutative. Otherwise we can find a non-trivial $g \in \operatorname{Ker}(\psi)$, so clearly $1-\varphi(g) \neq$ $0 \in \mathbb{K}(G)$. This shows that we can apply Theorem 3.1 which then concludes the proof.

In the case that $\varphi: \pi \rightarrow \pi / \pi_{r}^{(n+1)}$ we denote the semi-norm $\phi \mapsto \max \left\{0, \delta_{n}(\phi)\right\}$ by $\|-\|_{n}$. Note that in the case $n=0$ this was shown by the second author [7, Proposition 5.12] to be equal to McMullen's Alexander norm [11].

\subsection{Admissible triple}

We now slightly extend a definition from [8].

Definition Let $\pi$ be a group and $\psi: \pi \rightarrow \mathbb{Z}^{m}$ an epimorphism. Furthermore let $\varphi_{1}: \pi \rightarrow G_{1}$ and $\varphi_{2}: \pi \rightarrow G_{2}$ be epimorphisms to locally indicable and amenable groups $G_{1}$ and $G_{2}$. We call $\left(\varphi_{1}, \varphi_{2}, \psi\right)$ an admissible triple for $\pi$ if there exist epimorphisms $\Phi: G_{1} \rightarrow G_{2}$ and $\psi_{2}: G_{2} \rightarrow \mathbb{Z}^{m}$ such that $\varphi_{2}=\Phi \circ \varphi_{1}$, and $\psi=\psi_{2} \circ \varphi_{2}$.

Note that $\left(\varphi_{i}, \psi\right), i=1,2$ are admissible pairs for $\pi$. Combining Theorem 4.2 with [4, Theorem 1.3] (cf also [8]) we get the following result.

Theorem 4.3 Let $M$ be a 3-manifold with empty or toroidal boundary. If $\left(\varphi_{1}, \varphi_{2}, \psi\right)$ is an admissible triple for $\pi_{1}(M)$ such that $\tau\left(M, \varphi_{2}\right) \neq 0$, then we have the following inequalities of semi-norms:

$$
\|-\|_{\tau\left(M, \varphi_{2}\right)} \leq\|-\|_{\tau\left(M, \varphi_{1}\right)} \leq\|-\|_{T} .
$$

In particular we have

$$
\|-\|_{0} \leq\|-\|_{1} \leq \cdots \leq\|-\|_{T}
$$

Let $M$ be a 3-manifold with empty or toroidal boundary and let $\phi \in H^{1}(M ; \mathbb{Z})$. Since $\delta_{n}(\phi) \in \mathbb{N}$ for all $n$ it follows immediately from Theorem 4.3 that there exists $N \in \mathbb{N}$ such that $\delta_{n}(\phi)=\delta_{N}(\phi)$ for all $n \geq N$. But we can in fact prove a slightly stronger statement, namely that there exists such an $N$ independent of the choice of $\phi \in H^{1}(M ; \mathbb{Z})$. 
Proposition 4.4 Let $M$ be a 3-manifold with empty or toroidal boundary. There exists $N \in \mathbb{N}$ such that $\delta_{n}(\phi)=\delta_{N}(\phi)$ for all $n \geq N$ and all $\phi \in H^{1}(M ; \mathbb{R})$.

Proof Write $\pi=\pi_{1}(M), \pi_{n}=\pi / \pi_{r}^{(n+1)}$ and $m=b_{1}(M)$. Let $\psi: \pi \rightarrow \mathbb{Z}^{m}$ be an epimorphism. Write $\left(\pi_{n}\right)_{\psi}=\operatorname{Ker}\left\{\psi: \pi_{n} \rightarrow \mathbb{Z}^{m}\right\}$. Now pick elements $t^{\alpha} \in \pi_{n}, \alpha \in \mathbb{Z}^{m}$ such that $\psi\left(t^{\alpha}\right)=\alpha$ and $t^{k \alpha}=\left(t^{\alpha}\right)^{k}$ for any $\alpha \in \mathbb{Z}^{m}, k \in \mathbb{Z}$. Consider the map $\mathbb{Z}[\pi] \rightarrow \mathbb{Z}\left[\pi_{n}\right] \rightarrow \mathbb{K}\left(\left(\pi_{n}\right)_{\psi}\right)\left(t_{1}, \ldots, t_{m}\right)$. We write $\tau_{n}=\tau\left(M, \mathbb{K}\left(\left(\pi_{n}\right)_{\psi}\right)\left(t_{1}, \ldots, t_{m}\right)\right)$. We can find $f_{n}, g_{n} \in \mathbb{K}\left(\left(\pi_{n}\right)_{\psi}\right) \in\left[t_{1}^{ \pm 1}, \ldots, t_{m}^{ \pm 1}\right]$ such that $\tau_{n}=f_{n} g_{n}^{-1}$.

Now let $H$ be a real vector space and $C \subset H$ a convex subset. Then $C$ defines a dual convex subset $d(C) \subset H^{*}=\operatorname{hom}(H, \mathbb{R})$. Under the canonical identification $\left(H^{*}\right)^{*}=H$ we have $d(d(C))=C$. We use $\psi$ to identify $H_{1}(M ; \mathbb{R})$ with $\mathbb{R}^{m}$. Let $f=\sum_{\alpha \in \mathbb{Z}^{m}} a_{\alpha} t^{\alpha} \in \mathbb{K}\left(\left(\pi_{n}\right)_{\psi}\right) \in\left[t_{1}^{ \pm 1}, \ldots, t_{m}^{ \pm 1}\right]$ and denote by $N(f)$ its Newton polytope, ie $N(f)$ is the convex hull of $\left\{\alpha \mid a_{\alpha} \neq 0\right\} \subset H_{1}(M ; \mathbb{R})$. Clearly $d(N(f)) \subset$ $\left(H_{1}(M ; \mathbb{R})\right)^{*}=H^{1}(M ; \mathbb{R})$ equals the norm ball of $\|-\|_{f}$. By the above discussion we see that $d\left(\|-\|_{f}\right)=N(f)$, in particular $d\left(\|-\|_{f}\right)$ has only integral vertices.

By the definition of $\delta_{n}=\|-\|_{\tau_{n}}=\|-\|_{f_{g} g_{n}^{-1}}$ it follows that

$$
d\left(\delta_{n}\right)+d\left(g_{n}\right)=d\left(\tau_{n}\right)+d\left(g_{n}\right)=d\left(f_{n}\right)
$$

where " + " denotes the Minkowski sum of convex sets. It is easy to see that this implies that $d\left(\delta_{n}\right)$ has only integral vertices.

Theorem 4.3 implies that there is a sequence of inclusions

$$
d\left(\delta_{0}\right) \subset d\left(\delta_{1}\right) \subset \cdots \subset d\left(\|-\|_{T}\right) .
$$

Since $d\left(\|-\|_{T}\right)$ is compact and since $d\left(\delta_{n}\right)$ has integral vertices for all $n$ it follows immediately that there exists $N \in \mathbb{N}$ such that $d\left(\delta_{n}\right)=d\left(\delta_{N}\right)$ for all $n \geq N$. This completes the proof of the proposition.

\section{Examples}

Before we discuss the Thurston norm of a family of links we first need to introduce some notation for knots. Let $K$ be a knot. We denote the knot complement by $X(K)$. Let $\phi: H_{1}(X(K)) \rightarrow \mathbb{Z}$ be an isomorphism. We write $\delta_{n}(K):=\delta_{n}(\phi)$. This agrees with the original definition of Cochran [1] for $n>0$ and if $\Delta_{K}(t)=1$, and it is one less than Cochran's definition otherwise.

In the following let $L=L_{1} \cup \cdots \cup L_{m}$ be any ordered oriented $m$-component link. Let $i \in\{1, \ldots, m\}$. Let $K$ be an oriented knot with $\Delta_{K}(t) \neq 1$ which is separated 
from $L$ by a sphere $S$. We pick a path from a point on $K$ to a point on $L_{i}$ and denote by $L \#_{i} K$ the link given by performing the connected sum of $L_{i}$ with $K$ (cf Figure 1). Note that this connected sum is well-defined, ie independent of the choice of the path. We will study the Thurston norm of $L \#_{i} K$.
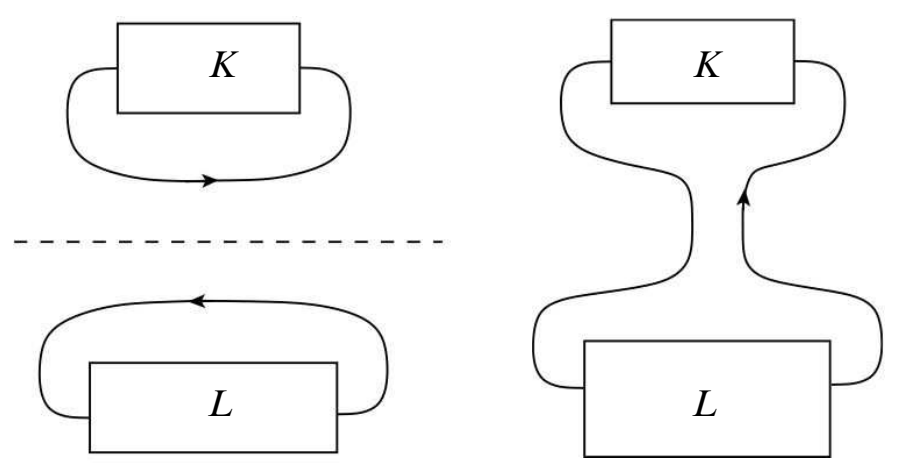

Figure 1: The link $L \#_{i} K$.

Now assume that $L$ is a non-split link with at least two components and such that $\|-\|_{0}=\|-\|_{T}$. Many examples of such links are known (cf [11]). For the link $L \#_{i} K$ denote its meridians by $\mu_{i}, i=1, \ldots, m$. Let $\psi: H_{1}\left(X\left(L \#_{i} K\right)\right) \rightarrow \mathbb{Z}^{m}$ be the isomorphism given by $\psi\left(\mu_{i}\right)=e_{i}$, where $e_{i}$ is the $i$ th vector of the standard basis of $\mathbb{Z}^{m}$.

We write $\pi:=\pi_{1}\left(X\left(L \#_{i} K\right)\right)$. For all $\alpha \in \mathbb{Z}^{m}$ we pick $t^{\alpha} \in \pi / \pi_{r}^{(n+1)}$ with $\psi\left(t^{\alpha}\right)=\alpha$ and such that $t^{l \alpha}=\left(t^{\alpha}\right)^{l}$ for all $\alpha \in \mathbb{Z}^{m}$ and $l \in \mathbb{Z}$. Furthermore write $t_{i}:=t^{e_{i}}$.

Proposition 5.1 Consider the natural map

$$
\varphi: \pi \rightarrow \mathbb{K}\left(\pi / \pi_{r}^{(n+1)}\right)=\mathbb{K}\left(\pi_{\psi} / \pi_{r}^{(n+1)}\right)\left(t_{1}, \ldots, t_{m}\right) .
$$

where $\pi$ is as defined above. There exists

$$
f\left(t_{i}\right) \in \mathbb{K}\left(\pi_{\psi} / \pi_{r}^{(n+1)}\right)\left[t_{i}^{ \pm 1}\right] \subset \mathbb{K}\left(\pi_{\psi} / \pi_{r}^{(n+1)}\right)\left[t_{1}^{ \pm 1}, \ldots, t_{m}^{ \pm 1}\right]
$$

such that $\operatorname{deg}\left(f\left(t_{i}\right)\right)=\delta_{n}(K)+1$, and there exists a $d=d\left(t_{1}, \ldots, t_{m}\right) \in \mathbb{K}\left(t_{1}, \ldots, t_{m}\right)$ with $\|-\|_{d}=\|-\|_{0}$, such that

$$
\tau\left(X\left(L \#_{i} K\right), \varphi\right)=d\left(t_{1}, \ldots, t_{m}\right) f\left(t_{i}\right) \in K_{1}\left(\mathbb{K}\left(\pi_{\psi} / \pi_{r}^{(n+1)}\right)\left(t_{1}, \ldots, t_{m}\right)\right) / \pm \varphi(\pi) .
$$

Furthermore, if $\delta_{n}(K)=2$ genus $(\mathrm{K})-1$, then

$$
\|-\|_{\tau\left(X\left(L \#_{i} K\right), \varphi\right)}=\|-\|_{T} .
$$


Proof Let $S$ be the embedded sphere in $S^{3}$ coming from the definition of the connected sum operation (cf Figure 1). Let $D$ be the annulus $S \cap X\left(L \#_{i} K\right)$ and we denote by $P$ the closure of the component of $X\left(L \#_{i} K\right) \backslash D$ corresponding to $K$. We denote the closure of the other component by $P^{\prime}$ (see Figure 2 below). Note that $P$ is homeomorphic to $X(K)$ and $P^{\prime}$ is homeomorphic to $X(L)$. Denote the induced

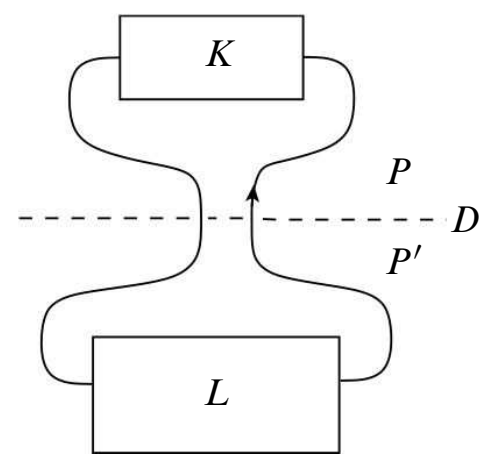

Figure 2: The link complement of $L \#_{i} K$ cut along the annulus $D$.

maps to $(K):=\mathbb{K}\left(\pi_{\psi} / \pi_{r}^{(n+1)}\right)\left(t_{1}, \ldots, t_{m}\right)$ by $\varphi$ as well. We get an exact sequence

$$
0 \rightarrow C_{*}^{\varphi}(D ;(K)) \rightarrow C_{*}^{\varphi}(P ;(K)) \oplus C_{*}^{\varphi}\left(P^{\prime} ;(K)\right) \rightarrow C_{*}^{\varphi}\left(X\left(L \#_{i} K\right) ;(K)\right) \rightarrow 0
$$

of chain complexes. It follows from [19, Theorem 3.4] that

$$
\tau(P, \varphi) \tau\left(P^{\prime}, \varphi\right)=\tau(D, \varphi) \tau\left(X\left(L_{i} \# K\right), \varphi\right) \in\left(K_{1}((K)) / \pm \varphi(\pi)\right) \cup\{0\} .
$$

First note that $D$ is homotopy equivalent to a circle and that $\operatorname{Im}\left\{\psi: \pi_{1}(D) \rightarrow \mathbb{Z}^{m}\right\}=$ $\mathbb{Z} e_{i}$. It is now easy to see that $\tau(D, \varphi)=\left(1-a t_{i}\right)^{-1}$ for some $a \in \mathbb{K}\left(\pi_{\psi} / \pi_{r}^{(n+1)}\right) \backslash\{0\}$. Next note that $\operatorname{Im}\left\{\psi: \pi_{1}(P) \rightarrow \mathbb{Z}^{m}\right\}=\mathbb{Z} e_{i}$. In particular $\tau(P, \varphi)$ is defined over the one-variable Laurent polynomial ring $\mathbb{K}\left(\pi_{\psi} / \pi_{r}^{(n+1)}\right)\left[t_{i}^{ \pm 1}\right]$ which is a PID. Recall that we can therefore assume that its Dieudonné determinant $f\left(t_{i}\right)$ lies in $\left.\mathbb{K} \pi_{\psi} / \pi_{r}^{(n+1)}\right)\left[t_{i}^{ \pm 1}\right]$ as well.

\section{Claim}

$$
\operatorname{deg}\left(\tau\left(P, \varphi: \pi_{1}(P) \rightarrow \mathbb{K}\left(\pi_{\psi} / \pi_{r}^{(n+1)}\right)\left(t_{i}\right)\right)=\delta_{n}(K) .\right.
$$

Proof First recall that there exists a homeomorphism $P \cong X(K)$. We also have an inclusion $X\left(L \#_{i} K\right) \rightarrow X\left(L_{i} \# K\right)$. Combining with the degree one map $X\left(L_{i} \# K\right) \rightarrow$ $X(K)$ we get a factorization of an automorphism of $\pi_{1}(X(K))$ as follows:

$$
\pi_{1}(X(K)) \cong \pi_{1}(P) \rightarrow \pi_{1}\left(X\left(L \#_{i} K\right)\right) \rightarrow \pi_{1}\left(X\left(L_{i} \# K\right)\right) \rightarrow \pi_{1}(X(K)) .
$$


Since the rational derived series is functorial (cf [7]) we in fact get that

$$
\begin{aligned}
\pi_{1}(X(K)) / \pi_{1}(X(K))_{r}^{(n+1)} & \cong \pi_{1}(P) / \pi_{1}(P)_{r}^{(n+1)} \\
& \rightarrow \pi_{1}\left(X\left(L_{i} \# K\right)\right)_{1} \pi_{1}\left(X\left(L_{i} \# K\right)\right)_{r}^{(n+1)} \\
& \rightarrow \pi_{1}(X(K)) / \pi_{1}(X(K))_{r}^{(n+1)}
\end{aligned}
$$

is an isomorphism. In particular

$$
\pi_{1}(X(K)) / \pi_{1}(X(K))_{r}^{(n+1)} \rightarrow \pi_{1}\left(X\left(L \#_{i} K\right)\right) / \pi_{1}\left(X\left(L \#_{i} K\right)\right)_{r}^{(n+1)}
$$

is injective, and the induced map on Ore localizations is injective as well. Finally note that $\operatorname{Ker}\left\{\pi_{1}(X(K)) \rightarrow \pi_{1}(P) \stackrel{\psi}{\rightarrow} \mathbb{Z}^{m}\right\}=\operatorname{Ker}(\phi)$ where $\phi: \pi_{1}(X(K)) \rightarrow \mathbb{Z}$ is the abelianization map. It now follows that

$$
\begin{aligned}
\delta_{n}(K) & =\operatorname{deg}\left(\tau\left(X(K), \pi_{1}(X(K)) \rightarrow \mathbb{K}\left(\pi_{1}(X(K))_{\phi} / \pi_{1}(X(K))_{r}^{(n+1)}\right)\left(t_{i}\right)\right)\right. \\
& =\operatorname{deg}\left(\tau\left(X(K), \pi_{1}(X(K)) \rightarrow \mathbb{K}\left(\pi_{\psi} / \pi_{r}^{(n+1)}\right)\left(t_{i}\right)\right)\right. \\
& =\operatorname{deg}\left(\tau\left(P, \pi_{1}(P) \rightarrow \mathbb{K}\left(\pi_{\psi} / \pi_{r}^{(n+1)}\right)\left(t_{i}\right)\right) .\right.
\end{aligned}
$$

Note that the second equality follows from the functoriality of torsion (cf [19, Proposition 3.6]) and the fact that going to a supfield does not change the degree of a rational function. This concludes the proof of the claim.

Claim We have the following equality of norms on $H^{1}(X(L) ; \mathbb{Z})$ :

$$
\|-\|_{\tau\left(P^{\prime}, \varphi\right)}=\|-\|_{T} .
$$

Proof First recall that $P^{\prime}$ is homeomorphic to $X(L)$. The claim now follows immediately from Theorem 4.3 applied to $\varphi$ and to the abelianization map of $\pi_{1}\left(P^{\prime}\right)$, and from the assumption that $\|-\|_{0}=\|-\|_{T}$ on $H^{1}(X(L) ; \mathbb{Z})$.

Putting these computations together and using Equation (3) we now get a proof of Equation (2).

Now assume that $\delta_{n}(K)=2$ genus $(\mathrm{K})-1$. Let $S_{i}$ be a Seifert surface of $K$ with minimal genus. Let $\phi: \mathbb{Z}^{m} \rightarrow \mathbb{Z}$ be an epimorphism and let $l=\phi\left(\mu_{i}\right) \in \mathbb{Z}$. We first view $\phi$ as an element in $\operatorname{hom}\left(H_{1}(X(L) ; \mathbb{Z})\right.$. A standard argument shows that $\phi$ is dual to a (possibly disconnected) surface $S$ which intersects the tubular neighborhood of $L_{i}$ in exactly $l$ disjoint curves. Then the connected sum $S^{\prime}$ of $S$ with $l$ copies of $S_{i}$ gives a surface in $X\left(L \#_{i} K\right)$ which is dual to $\phi$ viewed as an element in $\operatorname{hom}\left(H_{1}\left(X\left(L \#_{i} K\right) ; \mathbb{Z}\right)\right.$. A standard argument shows that $S^{\prime}$ is Thurston norm minimizing (cf eg [10, page 18]).

Clearly $\chi\left(S^{\prime}\right)=\chi(S)+l\left(\chi\left(S_{i}\right)-1\right)$. A straightforward argument shows that furthermore $\chi_{-}\left(S^{\prime}\right)=\chi_{-}(S)+l\left(\chi_{-}\left(S_{i}\right)+1\right)$ since $L$ is not a split link and since $K$ is non-trivial. 
We now compute

$$
\begin{aligned}
\|\phi\|_{T} & =\chi_{-}\left(S^{\prime}\right) \\
& =\chi_{-}(S)-n\left(\chi\left(S_{i}\right)-1\right) \\
& =\|\phi\|_{T}+2 l \operatorname{genus}(\mathrm{K}) \\
& =\|\phi\|_{d}+2\left(\delta_{n}(K)+1\right) \\
& =\|\phi\|_{d}+2 \operatorname{deg}\left(f\left(t_{i}\right)\right) \\
& =\|\phi\|_{\tau\left(X\left(L \#_{i} K\right), \varphi\right) .}
\end{aligned}
$$

By the $\mathbb{R}$-linearity and the continuity of the norms it follows that

$$
\|\phi\|_{\tau\left(X\left(L \#_{i} K\right), \varphi\right)}=\|\phi\|_{T}
$$

for all $\phi: \mathbb{Z}^{m} \rightarrow \mathbb{R}$.

We now combine Proposition 5.1 with results of [1] to give explicit examples of the sequence of semi-norms $\|-\|_{n}$.

Denote by $\diamond(n, m)$ the convex polytope given by the vertices $\left( \pm \frac{1}{n}, 0\right)$ and $\left(0, \pm \frac{1}{m}\right)$. Let $\left(n_{i}\right)_{i \in \mathbb{N}}$ and $\left(m_{i}\right)_{i \in \mathbb{N}}$ be never decreasing sequences of odd positive numbers which are eventually constant, ie there exists an $N$ such that $n_{i}=n_{N}$ for all $i \geq N$ and $m_{i}=m_{N}$ for all $i \geq N$. According to [1] we can find knots $K_{1}$ and $K_{2}$ such that $\delta_{i}\left(K_{1}\right)=n_{i}$ for any $i, \delta_{N}\left(K_{1}\right)=2 \operatorname{genus}\left(\mathrm{K}_{1}\right)-1$ and $\delta_{i}\left(K_{2}\right)=m_{i}$ for any $i$ and $\delta_{N}\left(K_{2}\right)=2 \operatorname{genus}\left(\mathrm{K}_{2}\right)-1$.

Let $H\left(K_{1}, K_{2}\right)$ be the link formed by adding the two knots $K_{1}$ and $K_{2}$ from above to the Hopf link (cf Figure 3). Recall that the Thurston norm ball of the Hopf link is given by $\diamond(1,1)$. Let $\pi:=\pi_{1}(X(L))$. It follows immediately from applying Proposition 5.1

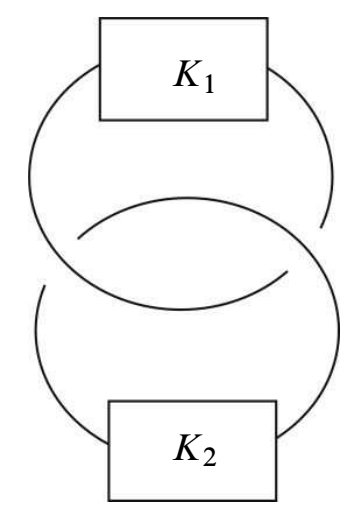

Figure 3: $H\left(K_{1}, K_{2}\right)$ is obtained by tying $K_{1}$ and $K_{2}$ into the Hopf link 
twice that the norm ball of $\|-\|_{i}$ equals $\diamond\left(n_{i}+1, m_{i}+1\right)$ and that $\|-\|_{N}=\|-\|_{T}$. The following result is now an immediate consequence of Proposition 5.1.

Corollary 5.2 We have the following sequence of inequalities of semi-norms

$$
\|-\|_{A}=\|-\|_{0} \leq\|-\|_{1} \leq\|-\|_{2} \leq \cdots \leq\|-\|_{N}=\|-\|_{T} .
$$

In [7] the second author gave examples of 3-manifolds $M$ such that

$$
\|-\|_{A}=\|-\|_{0} \leq\|-\|_{1} \leq\|-\|_{2} \leq \cdots
$$

but in that case it was not known whether the sequence of norms $\|-\|_{i}$ eventually agrees with $\|-\|_{T}$.

It is an interesting question to determine which 3-manifolds satisfy $\|-\|_{T}=\|-\|_{n}$ for large enough $n$. We conclude this paper with the following conjecture.

Conjecture 5.3 If $\pi_{1}(M)_{r}^{(\omega)}:=\bigcap_{n \in \mathbb{N}} \pi_{1}(M)_{r}^{(n)}=\{1\}$, then there exists $n \in \mathbb{N}$ such that $\|-\|_{T}=\|-\|_{n}$.

\section{References}

[1] T D Cochran, Noncommutative knot theory, Algebr. Geom. Topol. 4 (2004) 347-398 MR2077670

[2] T D Cochran, K E Orr, P Teichner, Knot concordance, Whitney towers and $L^{2}-$ signatures, Ann. of Math. (2) 157 (2003) 433-519 MR1973052

[3] J Dodziuk, $\mathbf{P}$ Linnell, $\mathbf{V}$ Mathai, T Schick, S Yates, Approximating $L^{2}-$ invariants and the Atiyah conjecture, Comm. Pure Appl. Math. 56 (2003) 839-873 MR1990479 Dedicated to the memory of Jürgen K Moser

[4] S Friedl, Reidemeister torsion, the Thurston norm and Harvey's invariants, Pacific J. Math. 230 (2007) 271-206

[5] S Friedl, T Kim, The parity of the Cochran-Harvey invariants of 3-manifolds, Trans. Amer. Math. Soc. (2005) In press

[6] S Friedl, T Kim, Twisted Alexander norms give lower bounds on the Thurston norm, Trans. Amer. Math. Soc. (2005) In press

[7] S L Harvey, Higher-order polynomial invariants of 3-manifolds giving lower bounds for the Thurston norm, Topology 44 (2005) 895-945 MR2153977

[8] S L Harvey, Monotonicity of degrees of generalized Alexander polynomials of groups and 3-manifolds, Math. Proc. Cambridge Philos. Soc. 140 (2006) 431-450 MR2225642 
[9] G Higman, The units of group-rings, Proc. London Math. Soc. (2) 46 (1940) 231-248 MR0002137

[10] W B R Lickorish, An introduction to knot theory, Graduate Texts in Mathematics 175, Springer, New York (1997) MR1472978

[11] C T McMullen, The Alexander polynomial of a 3-manifold and the Thurston norm on cohomology, Ann. Sci. École Norm. Sup. (4) 35 (2002) 153-171 MR1914929

[12] J Milnor, Whitehead torsion, Bull. Amer. Math. Soc. 72 (1966) 358-426 MR0196736

[13] D S Passman, The algebraic structure of group rings, Robert E. Krieger Publishing Co., Melbourne, FL (1985) MR798076 Reprint of the 1977 original

[14] J Rosenberg, Algebraic K-theory and its applications, Graduate Texts in Mathematics 147, Springer, New York (1994) MR1282290

[15] R Strebel, Homological methods applied to the derived series of groups, Comment. Math. Helv. 49 (1974) 302-332 MR0354896

[16] B Sturmfels, Solving systems of polynomial equations, CBMS Regional Conference Series in Mathematics 97, Published for the Conference Board of the Mathematical Sciences, Washington, DC (2002) MR1925796

[17] D Tamari, A refined classification of semi-groups leading to generalized polynomial rings with a generalized degree concept, from: "Proceedings of the International Congress of Mathematicians, Amsterdam, 1954", volume 3 (1957) 439-440

[18] W P Thurston, A norm for the homology of 3-manifolds, Mem. Amer. Math. Soc. 59 (1986) i-vi and 99-130 MR823443

[19] V Turaev, Introduction to combinatorial torsions, Lectures in Mathematics ETH Zürich, Birkhäuser Verlag, Basel (2001) MR1809561 Notes taken by Felix Schlenk

[20] V Turaev, A homological estimate for the Thurston norm (2002) arXiv: math.GT/0207267

[21] V Turaev, A norm for the cohomology of 2-complexes, Algebr. Geom. Topol. 2 (2002) 137-155 MR1885218

Department of Mathematics, Rice University

6100 Main Street, MS 136, Houston TX 77005, USA

Département de Mathématiques, UQAM

CP 8888, Succursale Centre-ville, Montréal, Qc H3C 3P8, Canada

shelly@math.rice.edu, friedl@alumni.brandeis.edu

Received: 18 August 2006

Algebraic 83 Geometric Topology, Volume 7 (2007) 